\title{
New Compactifications of Ten and Eleven Dimensional Supergravity on Manifolds Which Are Not Direct Products
}

\author{
P. van Nieuwenhuizen ${ }^{1, \star}$ and N. P. Warner ${ }^{2, \star \star}$ \\ 1 Institute for Theoretical Physics, State University of New York at Stony Brook, \\ Stony Brook, Long Island, NY 11794, USA \\ 2 California Institute of Technology, Pasadena, CA 91125, USA
}

\begin{abstract}
Solutions of ten and eleven dimensional supergravity are presented for which the space-time metric is a product of an anti-de Sitter metric and a strictly positive scalar function of the internal coordinates. The critical point of the potential of seven dimensional maximal supergravity with $\mathrm{SO}$ (4) symmetry is identified with such a solution.
\end{abstract}

In this letter we present new solutions of Kaluza-Klein supergravity for which the metric is of the form:

$$
g_{\hat{\mu} \hat{\nu}}(x, y, \alpha)=\left(\begin{array}{ccc}
\sigma^{2}(\alpha) g_{\mu \nu}(x) & 0 & 0 \\
0 & \varrho^{2}(\alpha) g_{\beta \gamma}(y) & 0 \\
0 & 0 & \tau^{2}(\alpha)
\end{array}\right) .
$$

The tensor $g_{\mu \nu}(x)$ is the metric for any Einstein space-time with positive cosmological constant and dimension $D_{1}$, and $g_{\beta \gamma}(y)$ is any Einstein metric with negative cosmological constant, describing $\left(D_{2}-1\right)$ dimensional internal space. The scaling functions $\varrho$ and $\sigma$ depend only upon the remaining internal coordinate, $\alpha$. To set our conventions: indices, $\beta, \gamma, \delta, \ldots$ and the coordinates $y^{\beta}$ refer to the $\left(D_{2}-1\right)$-dimensional internal space; $\mu, v, \varrho, \ldots$ and the coordinates $x$ refer to spacetime; $\hat{\mu}, \hat{v}, \hat{\varrho}, \ldots$ will refer to the entire $\left(D_{1}+D_{2}\right)$-dimensional space-time, and the coordinates $\left(x^{\mu}, y^{\beta}, \alpha\right)$ will be denoted generically by $z^{\hat{\mu}}$. The indices $a, b, c, \ldots$ and the coordinates $w^{a}$ will refer to the entire $D_{2}$-dimensional internal space, parametrized by $\left(y^{\beta}, \alpha\right)$.

The metric (1) no longer describes the direct product of a $D_{1}$-dimensional space-time with a $D_{2}$-dimensional internal space. Indeed, the curvature of spacetime depends on the internal space. However, it has already been shown [1], and we will demonstrate here, that such rescalings of the space-time metric are

* Supported in part by NSF Grant PHY 8109110 A-03

$\star \star$ Supported in part by the U.S. Department of Energy under Contract No. DE-AC-03-81ER40050, and by the Weingart Foundation 
necessary in order to obtain the scalar structure of the $D_{1}$-dimensional gauged supergravity theories by means of dimensional compactification.

The gauged supergravity theories have scalar potentials whose critical points provide one with backgrounds describing a $D_{1}$-dimensional Einstein space with positive cosmological constant and with some unbroken gauge symmetry, $H$. The metric is usually taken to be that of anti-de Sitter space-time. To obtain the corresponding solution in Kaluza-Klein supergravity, one starts by writing down the most general metric in $\left(D_{1}+D_{2}\right)$ dimensions which has anti-de Sitter invariance in the first $D_{1}$ dimensions,

$$
g_{\hat{\mu} \hat{v}}(z)=\left(\begin{array}{cc}
\sigma^{2}(w) g_{\mu v}(x) & 0 \\
0 & g_{a b}(w)
\end{array}\right),
$$

where $g_{\mu v}(x)$ is now the anti-de Sitter metric with unit radius. That is, its Riemann curvature is:

$$
R_{\mu \nu \varrho \sigma}(x)=-\left(g_{\mu \varrho} g_{v \sigma}-g_{\mu \sigma} g_{v \varrho}\right) .
$$

The metric $g_{a b}(w)$, and the function $\sigma^{2}(w)$ still have completely arbitrary dependence on the internal coordinates $w$. The existence of the extra degree of freedom, $\sigma^{2}(w)$, was already noted in [2], where it was also proved that (2) is the most general anti-de Sitter invariant metric. In [3] it was shown that the function $\sigma^{2}(w)$ was necessary to explain the vacua of the four-dimensional gauged $N=8$ theory [4] in terms of the $S^{7}$ compactification of eleven dimensional supergravity [5]. It has also been shown that for no choice of the function $\sigma(w)$, could the spacetime cosmological constant be made to vanish [6].

Recently, de Wit and Nicolai [1] found a solution of eleven dimensional supergravity with a non-trivial $\sigma^{2}(w)$ which provides a 4-dimensional space-time with $\mathrm{SO}(7)^{+}$symmetry. An analysis of the potential of $N=8$ supergravity [4], had revealed two solutions, one with $\mathrm{SO}(7)^{+}$and another with $\mathrm{SO}(7)^{-}$gauge symmetry [7, 8]. The latter seemed to correspond to the Englert solution [9]. The former critical point with $\mathrm{SO}(7)^{+}$symmetry was shown to correspond to the solution of reference [1]. This clearly demonstrates the importance of solutions with non-trivial $\sigma^{2}(w)$.

If $\sigma^{2}$ in (2) is constant, then the metric corresponds to that of a product manifold $M_{1} \times M_{2}$. On the other hand, suppose that $\sigma^{2}(w)$ vanishes at some point, then the metric becomes singular at this point. In general, to avoid real singularities at this point, topological identifications must be made, in which case the corresponding manifold is topologically different from $M_{1} \times M_{2}$. For example, consider the line element $d \theta^{2}+\left(1-\lambda \cos ^{2} \theta\right) \mathrm{d} \phi^{2}$. For $\lambda=0$ this is the metric on the 2-torus, whose Euler characteristic is zero. For $\lambda=1$ the coefficient of $d \phi^{2}$ vanishes at $\theta=0$ and $\theta=\pi$. This has the effect of pinching off the torus at each of these points and, of course the metric at $\lambda=1$ is that of the round 2-sphere, whose Euler characteristic is two.

All of the solutions we describe have non-vanishing $\sigma^{2}(w)$, and so the metrics may be continuously deformed back to ones which are products of $D_{1}$-dimensional internal space. In particular, our solutions may all be viewed as continuous deformations of some generalization of the Freund-Rubin ansatz. Accordingly, 
one might expect that the linearized field theory about these new solutions will decompose into spherical harmonics in much the same way as it does for $\sigma^{2} \equiv 1$.

We will present the following new solutions:

(i) Solutions of eleven dimensional supergravity compactifying to four dimensions, using any 6-dimensional Einstein space $g_{\beta \gamma}(y)$. This extends the solution of [1], which in our formulation corresponds to taking $g_{\beta \gamma}(y)$ to be the metric on the round $S^{6}$.

(ii) Solutions of eleven dimensional supergravity compactifying to a sevendimensional space-time, using a three-dimensional Einstein metric $g_{\beta \gamma}(y)$. In particular, there is only one solution which has an $\mathrm{SO}(4)$ internal symmetry, and it corresponds to the $\mathrm{SO}(4)$-invariant critical point $[10]$ of the potential of gauged maximal supergravity in seven dimensions [11].

(iii) Solutions of $N=2$, chiral supergravity in ten dimensions [12], compactifying to 5-dimensional space-time, using any 4-dimensional Einstein metric $g_{\beta \gamma}(y)$.

Our method for generating these solutions is quite general, and may obviously be used to find new solutions for other models.

The background metric will be taken to be of the form (1). The principle reason for using this specialized form of (2) is that it is obviously one of the simplest possible choices. It will be shown later that the solution of reference [1] may be reduced to a specific metric of the form (1). It is clear that the function $\tau$ is redundant in the parametrization of the metric, since one can choose a new coordinate $\beta(\alpha)$ such that $d \beta=\tau d \alpha$. However, should one use this parametrization, the resulting equations verge on the impossible. Our solutions would appear as complicated combinations of elliptic functions. The apparently redundant parameter, $\tau$, is extremely valuable in simplifying the form of our solutions.

For eleven dimensional supergravity we give the field $F_{\mu v \rho \sigma}$ the following expectation values:

$$
\begin{aligned}
D_{1}=7: F_{a b c d} & =m \sigma^{-D_{1}}\left[\operatorname{det} g_{a b}(w)\right]^{1 / 2} \varepsilon_{a b c d} \\
& =m \sigma^{-D_{1}} \tau \varrho^{3}\left[\operatorname{det} g_{\beta \gamma}(y)\right]^{1 / 2} \varepsilon_{a b c d}, \\
D_{1}=4: F_{\mu v \varrho \sigma} & =i m\left[\operatorname{det} g_{\mu v}(x)\right]^{1 / 2} \varepsilon_{\mu v \varrho \sigma},
\end{aligned}
$$

where $m$ is an arbitrary constant, and all other components of $F_{\mu v e \sigma}$ vanish. The Bianchi identities are obviously satisfied, and it is easy to check that the generalized Maxwell equations are automatically satisfied [this requires the factor of $\sigma^{-D_{1}}$ in (4)].

In the ten dimensional, chiral $N=2$ theory, the field equation for the four index antisymmetric tensor is that its curl is self-dual. Thus the most general anti-de Sitter invariant ansatz for this tensor field, with $D_{1}=5$, is:

$$
\begin{aligned}
F_{\mu \nu \sigma \sigma \lambda} & =i m\left[\operatorname{det} g_{\mu \nu}(x)\right]^{1 / 2} \varepsilon_{\mu \nu \varrho \sigma \lambda}, \\
F_{a b c d e} & =m\left[\operatorname{det} g_{a b}(w)\right]^{1 / 2} \varepsilon_{a b c d e} \sigma^{-5} \\
& =m \sigma^{-5} \varrho^{4} \tau\left[\operatorname{det} g_{\beta \gamma}(y)\right]^{1 / 2} \varepsilon_{a b c d e} .
\end{aligned}
$$

All other components of $F_{\mu \nu \rho \sigma}$, and all other fields, except the metric, vanish. It is straightforward to check that this ansatz is selfdual. 
It remains to solve the Einstein equations, with the sources (4)-(7). They are:

$$
\begin{gathered}
R_{\hat{\mu} \hat{v}}=-\frac{1}{6} F_{\hat{\mu} \hat{\varrho} \hat{\sigma} \hat{\tau}} F_{\hat{\nu}}^{\hat{\hat{\sigma}} \hat{\sigma}}+\frac{1}{72} g_{\hat{\mu} \hat{\nu}}\left(F_{\hat{\varrho} \hat{\sigma} \hat{\tau} \hat{\imath}}\right)^{2} \text { for } D_{1}+D_{2}=11, \\
R_{\mu \nu}=4 m^{2} \sigma^{-10} g_{\mu \nu}(x, y), R_{\alpha \beta}=-4 m^{2} \sigma^{-10} g_{a b}(y), \text { for } D_{1}+D_{2}=10 .
\end{gathered}
$$

We will first compute the Ricci tensor for the metric (1), with $D_{1}$ and $D_{2}$ arbitrary. Then we will make an ansatz based upon Weyl rescaling, to eliminate one of the functions $\varrho, \sigma$, and $\tau$. Finally, we shall consider the models discussed above and find a solution to the Einstein equations for these models. In what follows, we shall only assume that $g_{\mu \nu}(x)$ and $g_{\beta \gamma}(y)$ are Einstein metrics, and we shall not require (3).

Let

$$
E^{M}=\sigma(\alpha) e^{M}, E^{A}=\varrho(\alpha) e^{A}, E^{0}=\tau(\alpha) d \alpha,
$$

where $e^{M}$ and $e^{A}$ are the vielbeins for $g_{\mu v}(x)$ and $g_{\beta \gamma}(y)$. By taking exterior derivatives of (10) one obtains the spin connection $\Omega^{M N}$. Its components are

$$
\begin{aligned}
& \Omega^{M N}=\omega^{M N}\left(e^{M}\right) ; \Omega^{A B}=\omega^{A B}\left(e^{A}\right), \\
& \Omega^{M 0}=\sigma^{\prime} \sigma^{-1} \tau^{-1} E^{M} ; E^{A 0}=\varrho^{\prime} \varrho^{-1} \tau^{-1} E^{A},
\end{aligned}
$$

where capital letters denote flat indices and the superscript 0 denotes the flat index corresponding to the coordinate $\alpha$. Taking one more exterior deriative one obtains the curvatures

$$
R^{\hat{M} \hat{N}} \equiv \frac{1}{2} R^{\hat{M} \hat{N}}{ }_{\hat{P}} E^{\hat{P}} E^{\hat{Q}}=d \Omega^{\hat{M} \hat{N}}+\Omega^{\hat{M}}{ }_{\hat{K}} \wedge \Omega^{\hat{K} \hat{N}} .
$$

Normalizing the curvatures of $e^{M}$ and $e^{A}$ to

$$
R^{M N}{ }_{N P} \equiv R_{P}^{M}=\lambda_{1}\left(D_{1}-1\right) \delta_{P}^{M} ; R_{C}^{A} \equiv R_{B C}^{A B}\left(e^{A}\right)=-\lambda_{2}\left(D_{2}-2\right) \delta_{C}^{A},
$$

we find that the non-vanishing components of the Ricci tensor $R_{\hat{N}}^{\hat{M}_{\hat{N}}}$ are

$$
\begin{aligned}
R^{M}{ }_{N}= & \delta_{N}^{M} \tau^{-2}\left[\left(D_{1}-1\right)\left\{\tau^{2} \lambda_{1} \sigma^{-2}+\left(\sigma^{\prime} / \sigma\right)^{2}\right\}\right. \\
& \left.+\left(\sigma^{\prime \prime} / \sigma-\sigma^{\prime} \tau^{\prime} \sigma^{-1} \tau^{-1}\right)+\left(D_{2}-1\right) \sigma^{\prime} \varrho^{\prime} \sigma^{-1} \varrho^{-1}\right] \\
R_{B}^{A}= & \delta_{B}^{A} \tau^{-2}\left[-\left(D_{2}-2\right)\left\{\tau^{2} \lambda_{2} \varrho^{-2}-\left(\varrho^{\prime} / \varrho\right)^{2}\right\}\right. \\
& \left.+\left(\varrho^{\prime \prime} / \varrho-\varrho^{\prime} \tau^{\prime} \varrho^{-1} \tau^{-1}\right)+D_{1} \sigma^{\prime} \varrho^{\prime} \sigma^{-1} \varrho^{-1}\right] \\
R_{0}^{0}= & \tau^{-2}\left[D_{1}\left(\sigma^{\prime \prime} / \sigma-\sigma^{\prime} \tau^{\prime} \sigma^{-1} \tau^{-1}\right)\right. \\
& \left.+\left(D_{2}-1\right)\left(\varrho^{\prime \prime} / \varrho-\varrho^{\prime} \tau^{\prime} \varrho^{-1} \tau^{-1}\right)\right] .
\end{aligned}
$$

If $\tau^{2} \lambda_{2}=\sigma=1$ and $\varrho=\sin \alpha$, then, from (15) and (16), the internal metric must be that of a $D_{2}$-dimensional Einstein space. This we will consider as the "central" vacuum state, while the new vacua will be considered as a deformation of it. The compactification of eleven dimension supergravity on round $S^{7}$ and round $S^{4}$, and the compactification of ten dimension supergravity on the round $S^{5}$, would all be central vacua. If our new solutions are to be viewed as scalar deformations of the central vacuum, then one might expect the conformal rescaling, $\sigma$, of the spacetime metric to be equal to the Weyl rescaling suggested by compactification on flat 
manifolds $[13,14]$. Accordingly we take

$$
\sigma(\alpha)=C \Delta^{-\left(D_{1}-2\right)^{-1}}
$$

where $\Delta$ is the determinant of the vielbein deformations from the central vacuum. Clearly $\Delta=\tau \cdot\left(\frac{\varrho}{\sin \alpha}\right)^{\left(D_{2}-1\right)}$, and so we will eliminate $\varrho(\alpha)$ by taking

$$
\varrho(\alpha)=\left[\sigma(\alpha)^{D_{1}-2} \tau\right]^{-\left(D_{2}-1\right)^{-1}} \sin \alpha .
$$

The arbitrary constant in (17) has been absorbed into the arbitrary scalings, $\lambda_{1}$ and $\lambda_{2}$, of the Einstein spaces with metrics $g_{\mu \nu}$ and $g_{\beta \gamma}$.

In order to solve the Einstein equations, we begin with $R^{0}{ }_{0}$ in (16), from which we eliminate $\varrho^{\prime \prime} / \varrho$ and $\sigma^{\prime \prime} / \sigma$ by means of (14) and (15). From the result we eliminate $\varrho^{\prime}$ and $\varrho$ by means of (18). Denoting the Einstein equations in a vielbein frame generically by

$$
R_{0}^{0}=T_{1} ; R_{B}^{A}=\delta_{B}^{A} T_{2} ; R^{M}{ }_{N}=\delta_{N}^{M} T_{3},
$$

we get the following result

$$
\begin{aligned}
& \left(\frac{\sigma^{\prime}}{\sigma}\right)^{2}\left[2 D_{1}\left(D_{1}-2\right)-D_{1}\left(D_{1}-1\right)-\left(\frac{D_{2}-2}{D_{2}-1}\right)\left(D_{1}-1\right)^{2}\right] \\
& +\left(\frac{\tau^{\prime}}{\tau}\right)^{2}\left[-\left(\frac{D_{2}-2}{D_{2}-1}\right)\right]+\left(\frac{\sigma^{\prime} \tau^{\prime}}{\sigma \tau}\right)\left[2 D_{1}-2\left(\frac{D_{2}-2}{D_{2}-1}\right)\left(D_{1}-2\right)\right] \\
& +\left(\frac{\sigma^{\prime}}{\sigma}\right)\left[-2 D_{1}\left(D_{2}-1\right)+2\left(D_{1}-2\right)\left(D_{2}-2\right)\right] \cot \alpha \\
& +\left(\frac{\tau^{\prime}}{\tau}\right)\left[2\left(D_{2}-2\right)\right] \cot \alpha-\left(D_{2}-1\right)\left(D_{2}-2\right) \cot ^{2} \alpha
\end{aligned}
$$

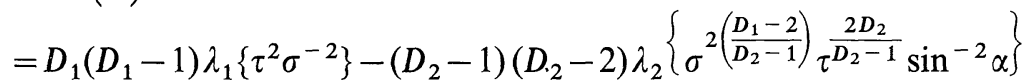

$$
\begin{aligned}
& -\tau^{2}\left\{D_{1} T_{3}+\left(D_{2}-1\right) T_{2}-T_{1}\right\}
\end{aligned}
$$

where we have multiplied throughout by $-\tau^{2}$.

Our strategy will now be as follows. The appearance of $\cot \alpha$ and $\sin ^{2} \alpha$ in (20) suggests that $\sigma$ and $\tau$ may well be simple functions of $\sin \alpha$, particularly since the $\sigma$ and $\tau$ independent term in (20) is proportional to $\cot ^{2} \alpha$. Guided by the discussion at the end of the paper about the solution of reference [1], we require that

$$
\sigma(\alpha)=a_{3}\left(1+l \sin ^{2} \alpha\right)^{m_{3}} ; \tau(\alpha)=a_{1}\left(1+l \sin ^{2} \alpha\right)^{m_{1}},
$$

for some constants $a_{1}, a_{3}, m_{1}, m_{3}$, and $l$. From (18) we find

$$
\varrho(\alpha)=a_{2}\left(1+l \sin ^{2} \alpha\right)^{m_{2}} \sin \alpha,
$$

where $a_{2}$ is a constant and

$$
m_{1}+\left(D_{2}-1\right) m_{2}+\left(D_{1}-2\right) m_{3}=0 .
$$

These choices ensure that the right-hand side of (20) takes a relatively simple form. Let $x \equiv \sin ^{2} \alpha$, then the terms bilinear in $\frac{\sigma^{\prime}}{\sigma}$ and $\frac{\tau^{\prime}}{\tau}$ in $(20)$ are proportional to 

$x(1-x)(1+l x)^{-2}$, whereas the terms linear in $\frac{\sigma^{\prime}}{\sigma}$ and $\frac{\tau^{\prime}}{\tau}$ in $(20)$ are proportional to
$(1-x)(1+l x)^{-1}$.

There are two terms with an $x^{-1}$ singularity in (20), and by requiring that the sum of their residues vanish, all terms on the left-hand side of (20) become, after multiplication by $(1+l x)^{2}$, proportional to either $x^{2}, x^{1}$ or $x^{0}$. Therefore, the righthand side of (20) must become a quadratic expression in $x$ after multipliation by $(1+l x)^{2}$. This will give three relations for the two exponents $m_{1}$ and $m_{3}$. The remaining exponent, $m_{2}$, will then be obtained from (23). Finally, the remaining constants will be determined (as far possible) from the Einstein Eqs. (14)-(16). The result will be a metric which satisfies all three equations completely. It should be noted that the scales $\lambda_{1}$ and $\lambda_{2}$ are redundant since they may be absorbed into $a_{2}$ and $a_{3}$. It is only the ratios $\lambda_{1} / a_{3}^{2}$ and $\lambda_{2} / a_{2}^{2}$ that will be determined.

For the compactification of eleven-dimensional supergravity to seven dimensions, the sources are determined by (4) and (8), and in the notation of (19) they are given by

$$
T_{3}=\frac{m^{2}}{3} \sigma^{-2 D_{1}} ; T_{1}=T_{2}=-\frac{2 m^{2}}{3} \sigma^{-2 D_{1}} .
$$

After multiplication by $(1+l x)^{2}$, the exponents of $(1+l x)$ for the three terms on the right-hand side of (20) are respectively

$$
\begin{aligned}
& N_{1}=2 m_{1}-2 m_{3}+2, \\
& N_{2}=\left(\frac{2 D_{2}}{D_{2}-1}\right) m_{1}+2\left(\frac{D_{1}-2}{D_{2}-1}\right) m_{3}+2, \\
& N_{3}=2 m_{1}-2 D_{1} m_{3}+2,
\end{aligned}
$$

where $D_{1}=7$ and $D_{2}=4$. However, the right-hand side must be quadratic in $x$. Hence $N_{1}, N_{2}$, and $N_{3}$ must be integers with

$$
0 \leqq N_{1}, N_{3} \leqq 2 ; 0 \leqq N_{2} \leqq 3 .
$$

One finds that there is only one possible solution

$$
m_{1}=m_{3}=\frac{1}{6} ; m_{2}=-\frac{1}{3} \text {. }
$$

The Einstein equations for (14) with source terms given by (19) and (24) are satisfied provided the coefficients of $x^{0}, x$, and $x^{2}$ vanish separately. This yields

$$
6\left(a_{1} / a_{3}\right)^{2} \lambda_{1}=1 ; l=-\frac{1}{2} ; m^{2} a_{1}^{2} a_{3}^{-14}=1 .
$$

The remaining two Einstein equations for (15) and (16) are then also satisfied provided the coefficients of the $x^{-1}$ terms as well as those of the $x^{0}, x$, and $x^{2}$ terms vanish. The vanishing of the $x^{-1}$ singularity in (15) forces the scale factors of $\varrho$ and $\tau\left(a_{2}\right.$ and $a_{1}$ respectively) to be the same as those for a $D_{2}$-sphere (for which $\varrho=\sin \alpha$ and $\tau$ is a constant), namely the square of their ratio must be equal to the curvature constant of the $\left(D_{2}-1\right)$-dimensional Einstein space

$$
\left(\frac{a_{1}}{a_{2}}\right)^{2} \lambda_{2}=1
$$

This relationship is model independent. 
One may check that with the choices of constants (27)-(29), all the Einstein equations are satisfied. Thus we have obtained the following solution of eleven dimensional supergravity, compactifying to seven dimension,

$$
\begin{aligned}
\sigma & =a_{3}\left(1-\frac{1}{2} \sin ^{2} \alpha\right)^{1 / 6} ; \tau=a_{1}\left(1-\frac{1}{2} \sin ^{2} \alpha\right)^{1 / 6}, \\
\varrho & =a_{2}\left(1-\frac{1}{2} \sin ^{2} \alpha\right)^{-1 / 3} \sin \alpha, \\
a_{3}^{-12} & =6 \lambda_{1} m^{-2} ;\left(\frac{a_{3}}{a_{1}}\right)^{2}=6 \lambda_{1} ;\left(\frac{a_{2}}{a_{1}}\right)^{2}=\lambda_{2} .
\end{aligned}
$$

Finally, we note that the Weyl rescaling condition (18) was only imposed in order to determine the ansatz (22), and the exponents in that ansatz (27). We then substituted the result directly into the Einstein equations and solved with an arbitrary coefficient $a_{2}$ in $\varrho(\alpha)$. If one imposes the conditions (18), one obtains the further constraint

$$
a_{1} a_{2}^{3} a_{3}^{5}=1 \Leftrightarrow \lambda_{2} m=\left(6 \lambda_{1}\right)^{11 / 3} .
$$

This sets the relative scale of the space-time and internal space in terms of the overall scale $m$.

As we remarked in the beginning of this paper, $\sigma(\alpha)$ does not vanish. Moreover, since $g_{\beta \gamma}(y)$ is a three-dimensional Einstein metric, it must be maximally symmetric, and therefore the round 3 -sphere (since it has negative curvature). Thus there is only one new solution, with $\mathrm{SO}(4)$ symmetry. The potential of maximal seven dimensional supergravity has two critical points [10], one with $\mathrm{SO}(5)$ symmetry, corresponding to a compactifiction on the round $S^{4}$ [15], and the other with $\mathrm{SO}(4)$ symmetry. We identify the second critical point with our new solution in (30). It would be interesting to see how the relative scales defined by (31), and the corresponding relative scales in the usual $S^{4}$ compactification, relate to the scales of the cosmological constants of the $\mathrm{SO}(4)$ and $\mathrm{SO}(5)$ invariant vacua of the seven dimensional gauged theory.

Next we apply our techniques to the compactification of eleven dimensional supergravity to four dimensions. From (8) and (5) one finds that

$$
T_{3}=-\frac{2 m^{2}}{3} \sigma^{-8} ; T_{1}=T_{2}=\frac{m^{2}}{3} \sigma^{-8}
$$

The same steps as before yield the solution

with

$$
\begin{aligned}
& \sigma=a_{3}\left(1-\frac{4}{5} \sin ^{2} \alpha\right)^{1 / 3} ; \tau=a_{1}\left(1-\frac{4}{5} \sin ^{2} \alpha\right)^{1 / 3}, \\
& \varrho=a_{2}\left(1-\frac{4}{5} \sin ^{2} \alpha\right)^{-1 / 6} \sin \alpha,
\end{aligned}
$$

$$
\left(\frac{a_{1}}{a_{3}}\right)^{2} \lambda_{1}=\frac{4}{3} ;\left(\frac{a_{1}}{a_{2}}\right)^{2} \lambda_{2}=1 ; m^{2} a_{1}^{2} a_{3}^{-8}=\frac{2}{5}, a_{1} a_{2}^{6} a_{3}^{2}=1 .
$$

Note the change of exponents in (33) in comparison to (30).

If $g_{\beta \gamma}(y)$ is the metric on the round six-sphere, we obtain the solution of reference [1], but, as we discussed before, we obtain a solution for any 6-dimensional compact Einstein space.

As another application of our method of obtaining solutions of supergravity models we consider the chiral $N=2, D=10$ supergravity theory [12]. It ia well- 
known that the field equations admit a solution which is the direct product of a five dimensional anti-de Sitter space-time and a round five sphere. Here we will construct a solution of the form (1), with $g_{\beta \gamma}(y)$ any 4-dimensional Einstein space with negative Ricci scalar. The Einstein Eqs. (19) lead via (16) and (20) to the same conditions as in (25), except that now $D_{1}=D_{2}=5$. Again there is a unique solution, for the same values of $N_{i}$, but the exponents $m_{1}$ are now given by

$$
m_{1}=m_{3}=-m_{2}=\frac{1}{4} \text {. }
$$

The first Einstein Eq. in (9), with (14) and (18) yields

$$
\left(\frac{a_{1}}{a_{3}}\right)^{2} \lambda_{1}=\frac{1}{2} ; l=-\frac{2}{3} ; m^{2} a_{1}^{2} a_{3}^{-10}=\frac{1}{12} .
$$

The remaining Einstein equations are satisfied, provided that (29) also holds. This leads to the solution

$$
\begin{aligned}
\sigma & =a_{1}\left(1-\frac{2}{3} \sin ^{2} \alpha\right)^{1 / 4} ; \tau=a_{3}\left(1-\frac{2}{3} \sin ^{2} \alpha\right)^{1 / 4}, \\
\varrho & =a_{2}\left(1-\frac{2}{3} \sin ^{2} \alpha\right)^{-1 / 4} \sin \alpha, \\
m^{2} a_{1} a_{3}^{-10} & =\frac{1}{12} ;\left(\frac{a_{3}}{a_{1}}\right)^{2}=2 \lambda_{1},\left(\frac{a_{2}}{a_{1}}\right)^{2}=\lambda_{2} .
\end{aligned}
$$

The imposition of the condition (18) on the scale factors $a_{1}, a_{2}$, and $a_{3}$ yields

$$
12 m^{2} \lambda_{2}^{2}=\left(2 \lambda_{1}\right)^{7 / 2} \text {. }
$$

It has been conjectured $[16,17]$ that the massless supermultiplet of the $S^{5}$ compactification of the chiral $N=2$ ten dimensional theory could be consistently truncated to a maximal $N=8$ gauged $\mathrm{SO}(6)$ supergravity theory in five dimensions. If this is so then presumably the solution (37), with $g_{\beta \gamma}$ taken to be the metric on the round 4-sphere, will correspond to an $\mathrm{SO}(5)$ invariant critical point of the scalar potential.

Although we have found only one solution of the triplet of second order partial differential equations, we expect that no further solutions exist which are smooth over the whole internal space.

Finally we describe the relationship of our work to that of reference [1], and show the motivation for our ansatz for $\varrho, \sigma$, and $\tau$.

The solution of [1] is defined as follows. Let $e_{\beta}^{b}(w)$ be the vielbein on the round $S^{7}$, and let $e_{\mu}^{m}(x)$ be the vielbein on the corresponding 4-dimensional anti-de Sitter space of the $S^{7}$ Freund-Rubin compactification. Let $\eta^{I}(w)$ be the Killing spinors on $S^{7}$ satisfying

$$
\left(\stackrel{\circ}{D}_{\beta}+\frac{i}{2} m_{7} \stackrel{o}{b}_{\beta}^{b} \Gamma_{b}\right) \eta^{I}(w)=0, \quad I=1, \ldots, 8 .
$$

Introduce the quantities

$$
\begin{aligned}
& \xi_{a}=\frac{i}{16} C_{I J K L} \bar{\eta}^{I} \Gamma_{a b} \eta^{J} \bar{\eta}^{K} \Gamma^{b} \eta^{L}, \\
& \xi=\frac{1}{16} C_{I J K L} \bar{\eta}^{I} \Gamma_{a} \eta^{J} \bar{\eta}^{K} \Gamma^{a} \eta^{L},
\end{aligned}
$$


where $C_{I J K L}$ is the $\mathrm{SO}(7)$ invariant, self-dual tensor of [8]. Let $\hat{\xi}_{a}$ denote the unit vector $\xi_{a}\left(\xi_{b} \xi^{b}\right)^{-1 / 2}$. Then the new $\mathrm{SO}(7)$ invariant solution of [1] has vielbein

where

$$
\begin{gathered}
e_{\mu}^{m}(x, w)=\Delta^{-1 / 2}(w) e_{\mu}^{m}(x), \\
e_{\beta}^{a}(w)=e_{\beta}^{b} S_{b a}(w),
\end{gathered}
$$

and $\tau$ is $a$ (constant) root of

$$
99 \tau^{2}+18 \tau-1=0 .
$$

Both roots produce the same metric.

First observe that $\xi_{\beta}=\frac{1}{2 m_{7}} \dot{D}_{\beta} \xi$. Moreover, $\xi$ has a much simpler form in terms of conformal scalars. Let $\phi^{i}(i=1, \ldots, 8)$ be the eight $k=1$ scalar modes of the Laplacian on $S^{7}$. These so-called conformal scalars satisfy a number of remarkable identities [18], which follow directly from the fact that if one embeds $S^{7}$ in $R^{8}$,one can identify the $\phi^{i}$ with the cartesian coordinates $x^{i}$. In particular we may normalize $\phi^{i}$ by

$$
\left.\phi^{i} \phi_{i}=1 \text { (sum over } i=1,8\right) \text {. }
$$

One can also show that (on a unit $S^{7}$ )

$$
\begin{aligned}
& \phi_{i} \dot{D}_{\beta} \phi^{i}=0, \\
& \grave{D}_{\beta}{\stackrel{\circ}{D_{\gamma}}}^{i} \phi^{i}=-g_{\beta \gamma} \phi^{i} \text {, }
\end{aligned}
$$

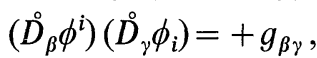

$$
\begin{aligned}
& \left(\check{D}_{\beta}^{\circ} \phi^{i}\right)\left(D^{\beta} \phi^{j}\right)+\phi^{i} \phi^{j}=\delta^{i j} .
\end{aligned}
$$

Furthermore the Killing vectors may be written as

$$
K_{\beta}^{i j}=\phi^{i} D_{\beta} \phi^{j}-\phi^{j} D_{\beta} \phi^{i},
$$

as well as in the form

$$
K_{\beta}^{I J}=\bar{\eta}^{I} \Gamma_{\beta} \eta^{J} .
$$

If one views the $\eta^{I}$ as transforming in the left-handed spinor representation of $\mathrm{SO}(8)$, then the $\phi^{i}$ transform in the vector representation of $\mathrm{SO}(8)$. However, selfdual tensors over left handed spinors are isomorphic as an $\mathrm{SO}(8)$ representation to symmetric tensors over the vectors. Therefore

$$
\begin{aligned}
\xi & =\frac{1}{16} C_{I J K L} \bar{\eta}^{I} \Gamma_{\beta} \eta^{J} \bar{\eta}^{K} \Gamma^{\beta} \eta^{L} \\
& =c \eta_{j k} K_{\beta}^{i j} K^{\beta i k},
\end{aligned}
$$

where $c$ is a constant depending upon normalization, and

$$
\eta_{i j}=\operatorname{diag}(1,1, \ldots, 1,-7)
$$


is the $\mathrm{SO}(7)$ invariant symmetric traceless tensor. Therefore, using (48) and (49) one obtains:

$$
\begin{aligned}
\xi & =\left(\sum_{i, j=1}^{8} K_{\beta}^{i j} K^{\beta i j}-2 K_{\beta}^{i 8} K^{\beta i 8}\right) \\
& =12 c\left[1-\phi^{2}\right]
\end{aligned}
$$

where $\phi=\phi^{8}=x^{8}$ is the eighth conformal scalar. In the polar coordinate system used at the outset of this paper we identify $x^{8}=\cos \alpha$ and hence $\xi=12 c \sin ^{2} \alpha$. Furthermore, it follows that

$$
\hat{\xi}_{a} d w^{a}=d \alpha
$$

and $g$ has the form

$$
g=\left(a+b \sin ^{2} \alpha\right)^{1 / 2},
$$

where $a$ and $b$ are constants. The precise ansatz (21) and (22), with exponents $m_{1}=m_{2}=1 / 3, m_{3}=-1 / 6$, follows directly from (41)-(47), (56), and (57). Observe that Eqs. (41) and (46) coincide with the condition (18).

At last it seems that we are understanding the ansatz for the scalars in relating massless sectors of the spherical compactifications to maximal supergravity theories in lower dimensions. In addition, this understanding has led us to a complete new class of solutions for any $g_{\beta \gamma}$ in an arbitrary Einstein metric with negative cosmological constant.

\section{References}

1. de Wit, B., Nicolai, H.: A new $\mathrm{SO}(7)$ invariant solution of $d=11$ supergravity. Phys. Lett. 148B, 60 (1984)

2. van Nieuwenhuizen, P.:De Witt, B.S., Stora, R. (eds.). Les Houches 1983 lectures, NorthHolland

3. Gunaydin, M., Warner, N.P.: The $G_{2}$ invariant compactifications of eleven dimensional supergravity. Caltech Preprint (1983) CALT-68-1077 (to appear in Nuc. Phys. B)

4. de Wit, B., Nicolai, H.: $N=8$ supergravity. Nucl. Phys. B 208, 323 (1982)

5. Duff, M.J.: In Supergravity 81. Ferrara, S., Taylor, J.G. (eds.). Cambridge: C.U.P. 1982

Duff, M.J., Pope, C.N.: In Supersymmetry and supergravity 82. Ferrara, S., Taylor, J.G., van Nieuwenhuizen, P. (eds.). Singapore: World Scientific Publishing 1983

Biran, B., Englert, F., de Wit, B., Nicolai, H.: Gauged $N=8$ supergravity and its breaking from spontaneous compactification. Phys. Lett. 124B, 45 (1983)

6. van Nieuwenhuizen, P., Kim, H.: To be published

7. Warner, N.P.: Some new extrema of the scalar potential of gauged $N=8$ supergravity. Phys. Lett. 128B, 169 (1983)

8. de Wit, B., Nicolai, H.: The parallelizing $S^{7}$ torsion in gauged $N=8$ supergravity. Nucl. Phys. B 231, 506 (1984)

9. Englert, F.: Spontaneous compactification of eleven-dimensional supergravity. Phys. Lett. 119B, 339 (1982)

10. Pernici, M., Pilch, K., van Nieuwenhuizen, P., Warner, N.P.: Non-compact gaugings and critical points of maximal supergravity in seven dimensions. Stony Brook preprint (1984), ITP-SB-84-41. Nucl. Phys. (to be published)

11. Pernici, M., Pilch, K., van Nieuwenhuizen, P.: Gauged maximally extended supergravity in seven dimensions. Phys. Lett. 143B, 103 (1904) 
12. Schwarz, J.H.: Covariant field equations of chiral $N=2, D=10$ supergravity. Nucl. Phys. B 226, 269 (1983)

13. Cremmer, E., Julia, B.: The SO (8) supergravity. Nucl. Phys. B 159, 141 (1979)

14. Scherk, J., Schwarz, J.H.: How to get masses from extra dimensions. Nucl. Phys. B 153, 61 (1979)

15. Pilch, K., Townsend, P.K., van Nieuwenhuizen, P.: Compactification of $d=11$ supergravity on $S^{5}$ (or $11=7+4$, too). Nucl. Phys. B 242, 377 (1984)

16. Gunaydin, M., Marcus, N.: The spectrum of the $S^{5}$ compactifition of the chiral $N=2, D=10$ supergravity and unitary supermultiplets of $U(2,2 / 4)$. Caltech preprint (1984), CALT-68-1185

17. Kim, H., Romans, L., van Nieuwenhuizen, P.: Stony Brook preprint ITP-SB-84-87

18. Gunaydin, M., Warner, N.P.:Spectrum generating algebras in Kaluza-Klein theories. Caltech preprint (1984), CALT-68-1151. Phys. Lett. B (to appear)

Communicated by S. W. Hawking

Received November 13, 1984 
